\title{
Mapping Accumulated Charges at the Semiconductor/Insulator Interface of Organic Field-Effect Transistors by Sum-Frequency Generation Spectroscopy*
}

\author{
Haiya Yang and Takayuki Miyamae ${ }^{\dagger}$ \\ Nanomaterials Research Institute, National Institute of Advanced Industrial Science and Technology (AIST), \\ 1-1-1 Higashi, Tsukuba, Ibaraki 305-8565, Japan \\ Masato Miyashita \\ Research Association of High-Throughput Design and Development for Advanced Functional Materials (ADMAT), \\ 1-1-1 Higashi, Tsukuba, Ibaraki 305-8565, Japan
}

(Received 8 January 2018; Accepted 22 July 2018; Published 9 August 2018)

\begin{abstract}
Charges accumulated at the semiconductor/insulator interface of a top-contact bottom-gate organic field-effect transistors (OFET) with a channel length/width of $1000 \mu \mathrm{m} / 1000 \mu \mathrm{m}$ while applying a negative gate voltage are visualized in a probe region of $4 \mathrm{~mm} \times 4 \mathrm{~mm}$ by electric-field induced sum-frequency generation spectroscopy. It is found the charges are accumulated not only inside but also outside the channel of the OFET. The accumulated charges are also found to be uniformly distributed on the semiconductor/insulator interface. The resolution of this mapping technique is explored to be $0.34 \mathrm{~mm}$ in the horizontal direction and $0.32 \mathrm{~mm}$ in the vertical. [DOI: 10.1380/ejssnt.2018.364]
\end{abstract}

Keywords: Organic field-effect transistors; Charge accumulations; Sum-frequency generation spectroscopy; Electric field; Semiconductor/insulator interface

\section{INTRODUCTION}

Due to their widespread applications in industry such as displays and imagers, organic field-effect transistors (OFET) have been interesting topics since 30 years ago [1]. Since the semiconductor/insulator interface is close to the conducting channel where accumulated charges are driven from source to drain electrodes during operation, charge transport is highly dependent on the quality of the semiconductor/insulator interface of OFET [2]. Secondharmonic generation (SHG) [3] and sum-frequency generation (SFG) [4] are intrinsically and specifically surfacesensitive techniques to probe molecular interactions on surfaces and buried interfaces where the inversion symmetry is necessarily broken. Electric field is required to be designedly induced into SHG and SFG while studying interfaces of OFET during operation. Electric-field induced SHG [5, 6] and SFG [7-9] are utilized to study interfaces of OFET and correlate their electrical properties and SHG or SFG measurements. Visualizing charge carriers in OFET during operation is an interesting and meaningful work among them, because this could help us monitor performances and clarify mechanisms related to charge transport of OFET. It is first successfully observed that charge carriers accumulated at the semiconductor/insulator interface of OFET fabricated with pentacene films and polyvinyl phenol dielectric layers while applying a negative gate voltage with an SFG microscope [8]. However, the probe region of the observed charge carriers in the SFG microscope is limited to $0.2 \mathrm{~mm} \times$ $0.2 \mathrm{~mm}$, which is too tiny to observe OFET samples for observation and investigation of the homogeneity of the interfaces of spin coating films.

In this study, electric-field induced SFG spectroscopy

\footnotetext{
* This paper was presented at the 8th International Symposium on Surface Science, Tsukuba International Congress Center, Tsukuba, Japan, October 22-26, 2017.

† Corresponding author: t-miyamae@aist.go.jp
}

is applied into observing charge accumulations and distributions at the semiconductor/insulator interface of OFET under operation, by mapping the position distributions of SFG intensity. It is found that during applying a negative gate voltage, the non-resonant background of the SFG intensity at the semiconductor/insulator interface of OFET increases substantially because of the increased electric field caused by positive charges accumulated at the semiconductor/insulator interface of OFET, while the SFG spectral shapes do not show any distinct changes at the same time. This increasing is found not only inside the conducting channel between source and drain electrodes, but also outside of them. The non-resonant SFG intensity shows an almost uniform increasing in the entire area of OFET, due to the charge accumulation. This work could help us clarify OFET mechanisms of charge accumulation during operation, and provide a guide about how to design OFET devices with much better performances. This work also extends the applications of SFG in the industry of organic electronics and facilitates the process of the development of this surface-detectable technique.

\section{EXPERIMENTAL}

\section{A. OFET}

An OFET sample is a typical top-contact bottomgate structure fabricated with two gold electrodes of $\sim 30 \mathrm{~nm}$ thick at the top and a semiconductor layer of 2,7-dioctyl[1]benzothieno[3,2-b]benzothiophene (C8BTBT) with a thickness of $\sim 50 \mathrm{~nm}$ below prepared through a spin-coating method (1000 rpm, $30 \mathrm{~s})$ with a C8-BTBT solution in chloroform (Wako, pure grade) of a concentration of $0.8 \mathrm{wt} \%$. A silicon substrate was used as a gate electrode with a $\mathrm{SiO}_{2}$ layer of $\sim 200 \mathrm{~nm}$ thick coated on its surface as an insulator. There are two types of channels in the OFET; one is length/width of $1000 \mu \mathrm{m} / 1000 \mu \mathrm{m}$, while the other is that of $500 \mu \mathrm{m} / 500 \mu \mathrm{m}$. A precision source/measure (Agilent, 
B2901A) was used to apply available gate voltages from $+100 \mathrm{~V}$ to $-100 \mathrm{~V}$ into OFET. The gate electrode is connected to high voltage end, whereas those two gold electrodes connected with each other are connected to ground.

\section{B. Sum-frequency generation spectroscopy}

An SFG spectrometer with a picosecond laser unit (Ekspla, PL2231-50) of $50 \mathrm{~Hz}$ repetition rate, a harmonic unit (Ekspla, SFGH500-2H), and an optical parametric generation unit (Ekspla, PG501-DFG1P) was used in this study to generate a visible beam at $532 \mathrm{~nm}$ and a tunable infrared (IR) beam ranging from $2.5 \mu \mathrm{m}$ to $10 \mu \mathrm{m}$, respectively. The polarization combination was set at PPP during measurements in which the IR, visible, and SFG beams were polarized in the plane of incidence. The incident angles for visible and IR beams were $65^{\circ}$ and $55^{\circ}$ from the surface normal, respectively. The output energies of lasers were reduced to the minimum possible level to avoid any damage on the sample. The step for the scanning of the $X Y$-maps was $0.2 \mathrm{~mm}$ for Fig. 3 and $0.01 \mathrm{~mm}$ for Fig. 4 for both $X$ and $Y$ directions using mechanical stepping motors. The SFG signal intensity was averaged by 100 times of acquisitions per point. All measurements were performed under ambient conditions. The temperature was kept at $20^{\circ} \mathrm{C}$ during all SFG experiments.

\section{RESULTS AND DISCUSSION}

\section{A. $\quad V_{\mathrm{g}}$ dependence on SFG spectra of OFET}

As shown in Fig. 1, we obtained SFG spectra of the channel region of OFET in PPP polarization combination during applying a series of gate voltages of $+40 \mathrm{~V}, 0 \mathrm{~V}$, and $-40 \mathrm{~V}$. In this experiment, because $V_{\mathrm{ds}}=0$, there is

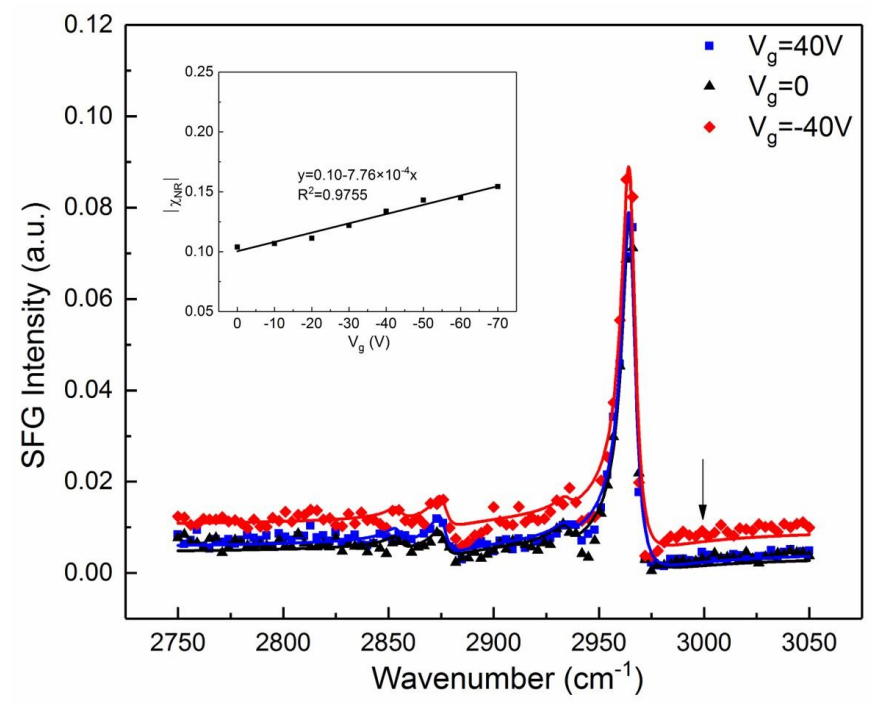

FIG. 1. The SFG spectra in PPP polarization combination taken at the center of the channel region of the OFET during applying a series of gate voltages of $+40 \mathrm{~V}, 0 \mathrm{~V}$, and $-40 \mathrm{~V}$. Each SFG spectrum is fitted based on Eq. (2); the inset shows a linear relationship of $\left|\chi_{\mathrm{NR}}\right|$ versus $V_{\mathrm{g}}$; the arrow pointed at $3000 \mathrm{~cm}^{-1}$ is discussed in the text of Sec. III C. no bias voltage between source and drain electrodes. Several SFG peaks observed in Fig. 1 should be derived from asymmetric and symmetric vibrations of alkyl chains of C8-BTBT in OFET. The SFG spectra under gate voltages of $0 \mathrm{~V}$ and $+40 \mathrm{~V}$ are almost mutually overlapped in which the strongest SFG peak at $2967 \mathrm{~cm}^{-1}$ should be assigned to the asymmetric stretch vibration of $\mathrm{CH}_{3}$. However, with applying a gate voltage of $-40 \mathrm{~V}$, the overall background of the SFG spectrum starts to increase obviously in contrast to those of $0 \mathrm{~V}$ and $+40 \mathrm{~V}$, although the strengths of SFG peaks themselves do not show such an apparent change.

That the overall background of the SFG spectra of OFET increases while applying a negative gate voltage, as well as its explanation have been comprehensively demonstrated elsewhere $[8,10]$. Here we only make a brief and supplemental explanation in this study by citing a related equation given by $[10-12]$

$$
P_{\mathrm{SFG}}=\chi^{(2)} E_{\mathrm{VIS}} E_{\mathrm{IR}}+\chi^{(3)} E_{\mathrm{VIS}} E_{\mathrm{IR}} E_{0},
$$

where $P_{\mathrm{SFG}}$ is nonlinear polarization of the SFG beam, $\chi^{(2)}$ and $\chi^{(3)}$ are the second- and third-order nonlinear susceptibilities, $E_{\mathrm{VIS}}$ and $E_{\mathrm{IR}}$ are electric fields of visible and IR beams, and $E_{0}$ is an applied DC electric field, respectively. Since C8-BTBT in OFET is a p-type semiconductor [13], hole carriers are injected and accumulate at the semiconductor/insulator interface under applying a negative gate voltage, which produces an electric-field effect there. So, the electric field of $E_{0}$ at this interface increases, resulting in an increasing of $\chi^{(3)} E_{\mathrm{VIS}} E_{\mathrm{IR}} E_{0}$ and thus leading to an increasing of $P_{\mathrm{SFG}}$. But such kind of carriers injection and accumulation do not happen as above if a reversely positive gate voltage is applied for a p-type semiconductor of C8-BTBT. This is the dominant reason for the unchanged SFG spectrum under the gate voltage of $+40 \mathrm{~V}$ shown in Fig. 1 as compared with that of $0 \mathrm{~V}$. The results of SFG spectra under different gate voltages of $-40 \mathrm{~V}, 0 \mathrm{~V}$, and $+40 \mathrm{~V}$ in Fig. 1 are exactly in accord with transfer characteristics of the OFET samples. In other words, when $V_{\mathrm{g}} \geq 0 \mathrm{~V}$, there is no drain current $I_{\mathrm{ds}}$ generated in p-typed OFET; but when $V_{\mathrm{g}}<0 \mathrm{~V}$, drain current $I_{\mathrm{ds}}$ would be generated there if absolute value of $V_{\mathrm{g}}$ exceeds that of threshold voltage $V_{\mathrm{th}}$, which is around $-20 \mathrm{~V}$ as we measured.

It is required to make a furthermore deeper discussion about $V_{\mathrm{g}}$ dependence on the measured SFG spectra of the OFET. The intensity of SFG output is given by [14]

$$
I_{\mathrm{SFG}} \propto\left|P_{\mathrm{SFG}}\right|^{2}=\left|\chi_{\mathrm{NR}}+\sum_{q} \frac{A_{q}}{\omega_{\mathrm{IR}}-\omega_{q}+i \Gamma_{q}}\right|^{2},
$$

where $\chi_{\mathrm{NR}}$ is non-resonant contribution to the susceptibility, $\omega_{\mathrm{IR}}$ is the frequency of IR beam, $A_{q}, \omega_{q}$, and $\Gamma_{q}$ are the amplitude, the frequency, and the damping constant of the $q$ th vibrational mode, respectively. Based on Eqs. (1) and (2), the nonlinear polarization of the SFG beam of $P_{\text {SFG }}$ consists of non-resonant contributions of $\chi_{\mathrm{NR}}$ and vibrationally resonant contributions of $\sum_{q} \frac{A_{q}}{\omega_{\mathrm{IR}}-\omega_{q}+i \Gamma_{q}}$ that are both supposed to be theoretically affected by the electric-field effect of $E_{0}$. The linear correlation of $\left|\chi_{\mathrm{NR}}\right|$ versus $V_{\mathrm{g}}$ that can be directly inferred 


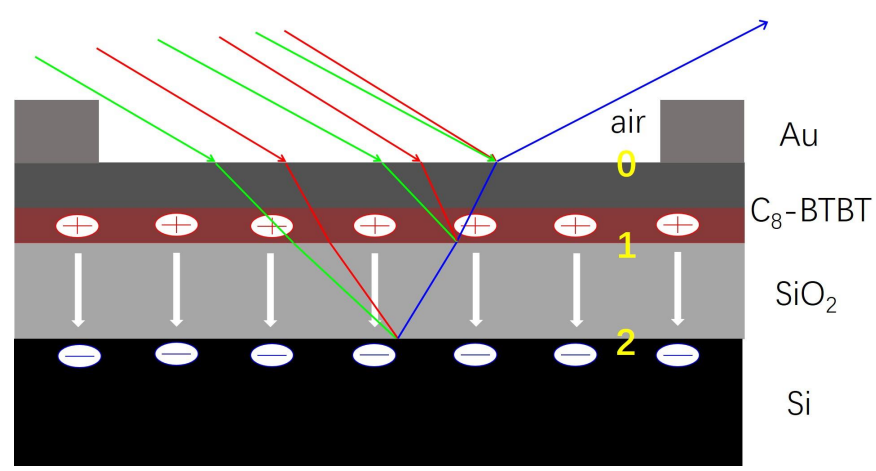

FIG. 2. A schematic illustration of three active interfaces of OFET probed by SFG spectroscopy and corresponding charge distribution.

from Eqs. (1) and (2) is experimentally evidenced by Ye et al. [10] and us as shown in the inset of Fig. 1. However, for vibrationally resonant contributions, different from the reported correlation between ratios of $\mathrm{CH}_{2} / \mathrm{CH}_{3}$ and $V_{\mathrm{g}}$ $[7,10]$, no outstanding changes in strengths of SFG peaks or ratios of $\mathrm{CH}_{2} / \mathrm{CH}_{3}$ are observed in our experiments shown in Fig. 1. This observation clearly indicates that there are no distinct orientation changes of alkyl chains of C8-BTBT in OFET under applying a negative gate voltage. We should note that the strengths of the vibrationally resonant SFG peaks do not show any changes by applying the voltage. This observation is different from the phenomenon observed in the electric field induced SFG taken from the organic light emitting diodes (OLED) under operation $[15,16]$. In the case of the OLED, the intensities of the vibrational peaks were also increased by applying the voltage, while such behavior was not found in C8-BTBT of OFET. Since the SFG measurement under the doubly-resonant condition was used for the measurement of the electric field induced SFG of the OLED, the intensities of the vibrational resonant SFG peaks were increased with the increase of the applied electric field. On the other hand, since the electronic transition of the alkyl chromophore is far from the visible wavelength region, and the current SFG measurements is not performed under doubly resonance condition, the electric field induced effect is observed just as an increase in the non-resonant background intensity.

Here we would like to discuss the contributions of measured SFG spectra in Fig. 1 from three different active interfaces in OFET. As shown in Fig. 2, since the OFET in this work has a multilayer structure with three interfaces from top to bottom, namely, the topmost air/semiconductor interface of 0 , the buried semiconductor/insulator interface of 1 , and the buried insulator/gate interface of 2, SFG signals could be generated from all of these three interfaces, including both resonant vibrations and non-resonant backgrounds from not only the interface 0 but also the interface 1 , and only non-resonant backgrounds from the interface 2 because there are no resonant vibrations coming from $\mathrm{SiO}_{2}$ at measured IR region [17-20]. The interface 1 is commonly regarded as the most important interface of OFET because it is the very place for charge accumulate and charge transport [21]. In reported work related to SFG measurements for OFET, the measured SFG signals are attributed to dominantly come from the interface 1 instead of the others, although the total SFG signals may include contributions from the interfaces 0 and 2 in terms of multiple interference effects $[8,10]$. In our experiment, it is consistently believed the measured SFG spectra and their $V_{\mathrm{g}}$ dependence should come from mainly the interface 1 , with our explanations shown as follows. While applying a negative gate voltage, injected holes ought to accumulate at the interface 1 and generate an increased electric field there. This makes the total background of SFG intensities to increase as explained early based on Eq. (1). But such movement of charge accumulation does not happen at the other two interfaces of 0 and 2. Injected holes do not go to the interface 0 and stay there under a negative gate voltage. Although electrons generated in the gate electrode at the same time because of injected holes accumulate at the interface 1 , and some of them certainly go to and stay at the interface 2 , they do not aggregate there as the same way as the injected holes at the interface 1 . In other words, the roles these electrons play on the electric field at the interface 2 are too weak to make a comparably large enhancing of non-resonant background there while applying the gate voltage, different from those of injected holes at the interface 1 . Consequently, while applying a gate voltage of $-40 \mathrm{~V}$, the increased total background of SFG intensity in Fig. 1 are mainly caused by the charge accumulation that could only happen at the interface 1.

\section{B. Charge accumulations in OFET mapped by sum-frequency generation spectroscopy}

In order to explore the position dependence of the SFG intensity on the semiconductor/insulator interface of OFET without and with applying a negative gate voltage, we show the in-plane SFG signal intensity distribution around the source and drain electrodes in Fig. 3. In this measurement, the wavelength of $3000 \mathrm{~cm}^{-1}$ is selected as a probe wavelength (see the arrow in Fig. 1). We scanned its SFG intensity around two gold electrodes and their surroundings including the channel with gate voltages of $0 \mathrm{~V}$ and $-80 \mathrm{~V}$ relative to the source and drain contacts $\left(V_{\mathrm{ds}}=0 \mathrm{~V}\right)$. In this experiment, the applied gate voltage is enhanced from $-40 \mathrm{~V}$ to $-80 \mathrm{~V}$ for observing more drastically increased SFG intensities in order to obtain an $X Y$-map with a sharper contrast, although $-40 \mathrm{~V}$ is selected to be the gate voltage to observe the electric-field effect in the SFG data taken in Fig. 1. Based on the SFG intensity at $3000 \mathrm{~cm}^{-1}$, an $X Y$-map of SFG intensity with and without applying a gate voltage of $-80 \mathrm{~V}$ is obtained. The wavelength of $3000 \mathrm{~cm}^{-1}$ is preferred to those of SFG peaks such as $2965 \mathrm{~cm}^{-1}$ because the SFG signals at $3000 \mathrm{~cm}^{-1}$ is dominantly contributed from nonresonant background, which has an exactly proved linear relationship with $V_{\mathrm{g}}$ [10]. For the wavelength of SFG peaks like $2965 \mathrm{~cm}^{-1}$, although resonant SFG signals are assumed to be also affected by the electric field, the intensities of resonant SFG signals change less radically as $V_{\mathrm{g}}$ varies $[7,10]$.

As shown in Fig. 3(a, b), charge accumulations at the semiconductor/insulator interface and the electric field between source and drain electrodes are obviously visualized by mapping the position distributions of SFG inten- 

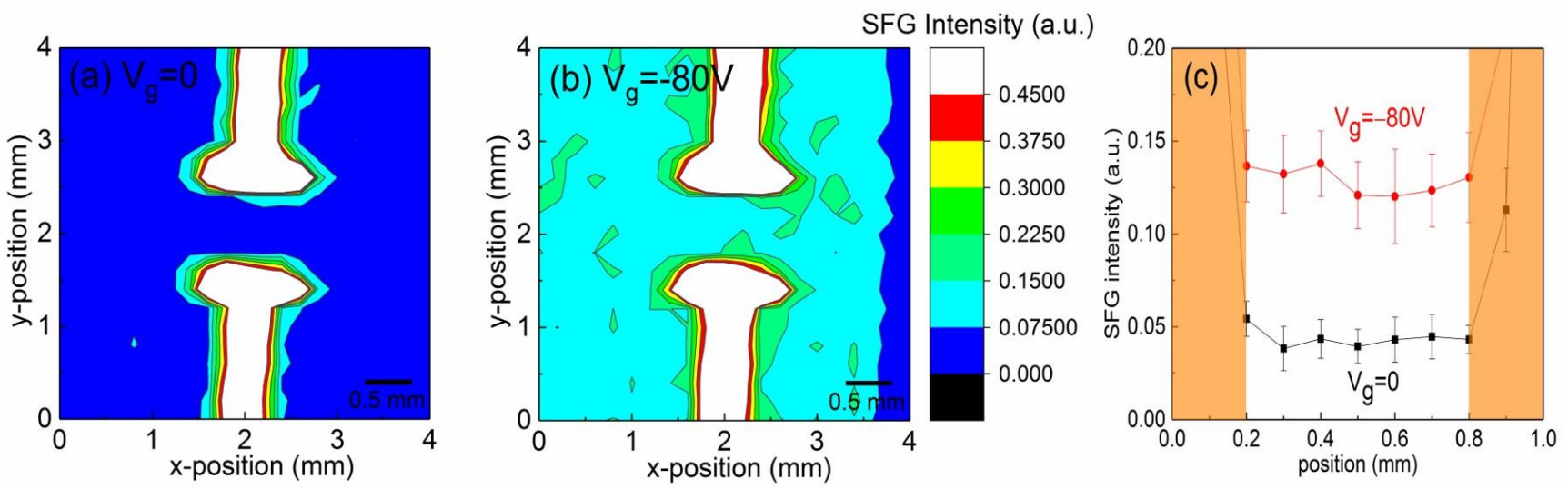

FIG. 3. The $X Y$-maps of the OFET surface based on the SFG intensities at the wavelength of $3000 \mathrm{~cm}^{-1}$ in PPP polarization combination without (a) and with (b) applying a gate voltage of $-80 \mathrm{~V}$ (c) the positon dependence from source to drain electrodes of SFG intensities at the wavelength of $3000 \mathrm{~cm}^{-1}$ without and with applying a gate voltage of $-80 \mathrm{~V}$.

sity at $3000 \mathrm{~cm}^{-1}$. This is a dot-by-dot scanning of SFG intensities at $3000 \mathrm{~cm}^{-1}$ in PPP polarization combination on the sample surface. The SFG intensities generated at the gold electrodes and C8-BTBT are largely different under the same experimental conditions, since the gold surfaces generate much stronger frequency-independent SFG signals than organic thin film interfaces [22]. Based on this significant difference, we can effortlessly locate the semiconductor layer, the gold electrodes, the channel region, and the region outside the channel, respectively. Because SFG intensity is plotted against $X$ and $Y$ positions, the colors in this $X Y$-map will change drastically once there is any change on the SFG intensities in this area. Without applying a negative gate voltage into the OFET, the white region is considered to be gold electrodes, whereas the blue regions outside the white region is the semiconductor layer. The SFG signal intensities seems to be uniformly distributed. The absence of intensity variations in the plane of the semiconductor area indicates that the spin-coating film is almost uniform at least within the measured area. While applying a gate voltage of $-80 \mathrm{~V}$, the blue region changes into cyan not only inside the channel but also outside the channel, which means the SFG intensity there increased under applying the gate voltage in contrast to that without applying the negative gate voltage. The reason for the increased SFG intensity has been explained in Sec. III A that positive charges accumulated at the semiconductor/insulator interface.

Charge accumulations distributed homogenously around source and drain electrodes in a region of $4 \mathrm{~mm} \times$ $4 \mathrm{~mm}$ are first mapped out by electric-field induced SFG spectroscopy as shown in Fig. 3(a, b). It provides us an additional way of electric-field induced SFG spectroscopy to visualize accumulated charge carriers and electric-field distributions at the semiconductor/insulator interface in OFET in a much larger probe region, as well as in OFET with much longer and wider channels than typical ones with a channel length/width of $95 \mu \mathrm{m} / 145 \mu \mathrm{m}$ [8]. OFET mechanisms related to charge carriers could also be clarified by such kind of visualizations, which would provide a guidance for designing new ones to improve performances of OFET.

One big difference in this $X Y$-map from that of Mat- sumoto's group [8] is there are some color changes even outside the conducting channel. This probably depends on the construction of OFET instead of SFG techniques. In their OFET configuration, the gold electrodes might be embedded into the semiconductor and blocked the channel from its outside region. But in our case, the gold electrodes are actually paved on the semiconductor, and the entire semiconductor layer beneath the gold electrodes could be the channel. So, while applying a negative gate voltage, the color of the outside region of the channel also changes into cyan, which means charges are also accumulated here. Surely, mapping the OFET surface only by SFG intensity generates some minor errors at the same time. For example, before and after applying $V_{\mathrm{g}}$, the shape of gold electrodes seems to have some slight changes, as shown in Fig. 3(a, b), but this could not happen in fact. In any case, this $X Y$-map by SFG intensity scanned with SFG spectroscopy could help us accurately locate the source and drain electrodes, the semiconductor layer, and the conducting channel conveniently.

Another obvious difference from the result of SFG microscopy is that accumulated charges are almost uniformly distributed from drain to source electrodes. In order to illustrate this situation more clearly, Fig. 3(c) is plotted based on the result of the channel region in Fig. 3(a, b). As shown in Fig. 3(c), the changes in the curve of $V_{\mathrm{g}}=-80 \mathrm{~V}$ from that of $V_{\mathrm{g}}=0 \mathrm{~V}$ are within errors of them, which illustrates that accumulated charges are almost uniformly distributed from drain to source electrodes, and even the entire surface of the semiconductor of OFET. Here we make an explanation about this homogenous distribution of charges at the interface. Before applying the gate voltage $V_{\mathrm{g}}$, the OFET is in the deplete state; after adding $V_{\mathrm{g}}$, it is in the accumulated state. At this time, $V_{\mathrm{ds}}=0 \mathrm{~V}$, so there is no bias voltage between source and drain electrodes, which means the electric field between these two electrodes should be homogenous distributed. However, it could be expected that when applying a strong bias voltage $V_{\mathrm{ds}}$ between source and drain electrodes of OFET during operation, there would be an expected bias position dependence of SFG intensity between source and drain electrodes caused by the diagonal distribution of the quantity of charge carriers between two electrodes. This will be our future work. 

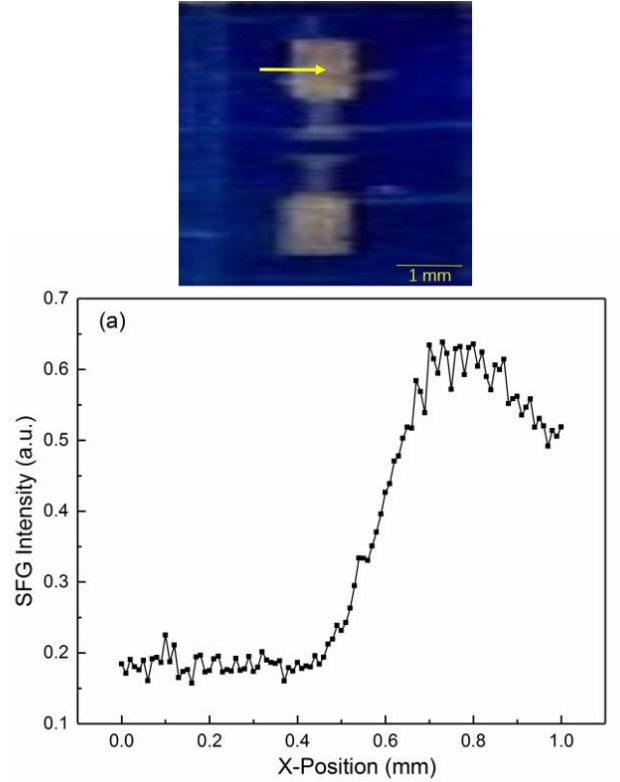
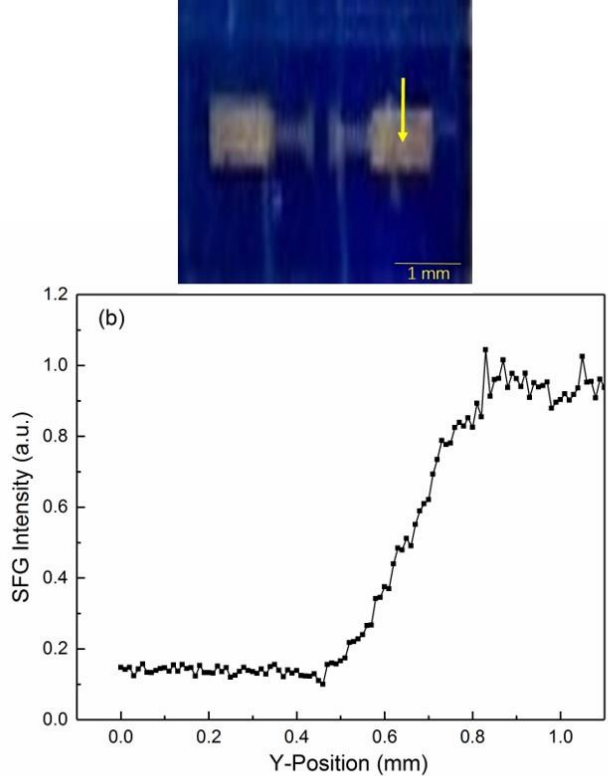

FIG. 4. The $X$-position and $Y$-position dependences from C8-BTBT to the gold electrode of SFG intensities at the wavelength of $3000 \mathrm{~cm}^{-1}$ in PPP polarization combination. Corresponded photos of the scanning processes on gold electrodes of OFET are also shown above.

\section{The resolution in the $X Y$-map of sum-frequency generation spectroscopy}

The SFG intensities obtained by SFG spectroscopy are utilized to map the charge accumulations at the semiconductor/insulator interface of OFET; Therefore, we have to illustrate the resolution of this mapping technique of SFG. The same method as that in the SFG microscopy imaging is selected to calculate the horizontal and vertical resolution for the SFG spectroscopy during its mapping process shown in Fig. 4, in which the SFG beam is allowed to scan a region consisted of two flats with extremely different SFG intensities horizontally and vertically. It is considered that the distance between $10 \%$ and $90 \%$ intensity is the resolution for both horizontal and vertical [23]. As shown in Fig. 4, the resolution in our technique is evaluated to be $340 \mu \mathrm{m}$ for the horizontal and $320 \mu \mathrm{m}$ for the vertical edge of the gold electrode. Although this resolution is still too large to see the local defects or charge traps inside the OFET channel, especially far from the smallest one of $0.48 \mu \mathrm{m}$ for a sum-frequency generation confocal microscope reported [24], it is enough for us to probe a mapped region of $4 \mathrm{~mm} \times 4 \mathrm{~mm}$ of the semiconductor/insulator interface of OFET, which indicates that our conclusion is reasonable and convincing. It is feasible to furthermore improve the resolution of the mapping of SFG spectroscopy evaluated in this kind of experiment in our future work.

\section{CONCLUSIONS}

In this work, SFG spectroscopy is utilized to observed charge accumulations occurring at the semiconductor/insulator interface of OFET during the application of a negative gate voltage. It is found that charge accumulations are mainly distributed around the source and drain electrodes not only inside the channel between source and drain electrodes but also outside the channel, and this SFG signals almost homogenous distribute everywhere especially between gold electrodes, which reflects the electric field is homogenously distributed around the channels. The resolution of this mapping technique is also explored and calculated to be $340 \mu \mathrm{m}$ for the horizontal and $320 \mu \mathrm{m}$ for the vertical edge. The accumulated charges and the electric field around the electrodes in OFET are visualized by spectroscopic SFG technique and its mapping. This work adds a new electric-field induced SFG spectroscopic way to observe uniformity/nonuniformity distribution of the accumulated charges in OFET, and help us clarify OFET mechanisms related to charges and the deciding factors of the performances of OFET. This work extends the applications of SFG in the industry of organic electronics, and will facilitate the process of the development of this surface-detectable technique.

\section{ACKNOWLEDGMENTS}

This study is supported by New Energy and Industrial Technology Development Organization (NEDO) through a commissioned project (P16010).
[1] I. Kymissis, Organic Field Effect Transistors: Theory, Fabrication and Characterization (Springer, Boston, 2009).
[2] G. Horowitz, in: Organic Electronics. Advances in Polymer Science, Vol. 223, edited by T. Grasser, G. Meller, and L. Li (Springer, Berlin, Heidelberg, 2009) p. 113. 
[3] Y. R. Shen, Annu. Rev. Phys. Chem. 40, 327 (1989).

[4] Y. R. Shen, Nature 337, 519 (1989).

[5] D. Yamada, T. Manaka, E. Lim, R. Tamura, M. Weis, and M. Iwamoto, J. Appl. Phys. 104, 074502 (2008).

[6] T. Manaka, F. Liu, M. Weis, and M. Iwamoto, J. Phys. Chem. C 113, 10279 (2009).

[7] H. Ye, A. Abu-Akeel, J. Huang, H. E. Katz, and D. H. Gracias, J. Am. Chem. Soc. 128, 6528 (2006).

[8] I. F. Nakai, M. Tachioka, A. Ugawa, T. Ueda, K. Watanabe, and Y. Matsumoto, Appl. Phys. Lett. 95, 243304 (2009).

[9] T. C. Anglin, D. B. O'Brien, and A. M. Massari, J. Phys. Chem. C 114, 17629 (2010).

[10] H. Ye, J. Huang, J.-R. Park, H. E. Katz, and D. H. Gracias, J. Phys. Chem. C 111, 13250 (2007).

[11] D. S. Bethune, R. W. Smith, and Y. R. Shen, Phys. Rev. Lett. 38, 647 (1977).

[12] D. E. Gragson and G. L. Richmond, J. Phys. Chem. B 102, 3847 (1998).

[13] H. Ebata, T. Izawa, E. Miyazaki, K. Takimiya, M. Ikeda, H. Kuwabara, and T. Yui, J. Am. Chem. Soc. 129, 15732 (2007).

[14] T. Miyamae, A. Morita, and Y. Ouchi, Phys. Chem.
Chem. Phys. 10, 2010 (2008).

[15] T. Miyamae, N. Takada, and T. Tsutsui, Appl. Phys. Lett. 101, 073304 (2012).

[16] T. Miyamae, N. Takada, H. Ohata, and T. Tsutsui, Appl. Phys. Express 10, 102101 (2017).

[17] Y. Tong, Y. Zhao, N. Li, M. Osawa, P. B. Davies, and S. Ye, J. Chem. Phys. 133, 034704 (2010).

[18] X. Lu, M. L. Clarke, D. Li, X. Wang, G. Xue, and Z. Chen, J. Phys. Chem. C 115, 13759 (2011).

[19] D. B. O'Brien and A. M. Massari, J. Chem. Phys. 138, 154708 (2013).

[20] D. B. O'Brien and A. M. Massari, J. Chem. Phys. 142, 024703 (2015).

[21] H. Dong, L. Jiang, and W. Hu, Phys. Chem. Chem. Phys. 14, 14165 (2012).

[22] M. Himmelhaus, F. Eisert, M. Buck, and M. Grunze, J. Phys. Chem. B 104, 576 (2000).

[23] D. M. P. Hoffmann, K. Kuhnke, and K. Kern, Rev. Sci. Instrum. 73, 3221 (2002).

[24] N. A. Tuan, Y. Miyauchi, and G. Mizutani, Jpn. J. Appl. Phys. 51, 122402 (2012). 